\title{
Dominant Inherited Tilted Disc Syndrome and Lacquer Cracks
}

\author{
F. G. BOTTONI*, C. A. EGGINK**, J. R. M. CRUYSBERG**, A. M. VERBEEK ${ }^{* *}$ \\ Milan, Italy and Nijmegen, The Netherlands
}

\begin{abstract}
Summary
Three patients with the tilted disc syndrome from one family were examined. The presence of the trait in three consecutive generations suggests an autosomal dominant mode of inheritance, although in these patients with variable expression.

The propositus showed bilateral inferonasal retinal ectasia, with atrophic subretinal scars. Linear-like lacquer cracks, radiating from the central scars, were also present running parallel to the margin of the optic nerve head. The linear streaks were very similar to those usually seen in traumatic tears of Bruch's membrane.

The mechanical stretching of the ectatic area, and its abnormal location inferonasal to the optic disc might have been responsible for the unusual pattern of the lacquer cracks in our patient.
\end{abstract}

The tilted disc syndrome is a condition characterised by

(1) subnormal vision,

(2) myopic astigmatism with oblique cylinders,

(3) transposition (tilting) of the optic disc downward and nasally,

(4) inferior or inferonasal crescent (congenital conus),

(5) nasal or inferonasal ectasia associated with thinning of the pigment epithelium and choroid,

(6) (bilateral) temporal loss of visual field. ${ }^{1}$

The wide spectrum of changes observed in the syndrome might depend upon the degree of vigour of closure of the embryonic fissure at the six-week stage of gestation. The spectrum ranges from a minimal defect (tilted disc) to more severe fundus involvement (i.e., inferonasal ectasia, frank coloboma). ${ }^{1}$

In some opinions, this assumption does not explain adequately the origin of all ocular anomalies found in the syndrome. Fewer retinal nerve fibres than normal enter the defective side of the disc. ${ }^{2}$ Therefore, it has been suggested $^{3}$ that atrophy of the ganglion cell fibres of the inferonasal retina might occur following an anomalous closure of the embryonic cleft. This in turn would explain the hypoplasia of the inferonasal quadrant, the inferior crescent (lack of fibres in the inferior optic disc) and the anomalous appearance of the optic nerve head and of the retinal vessels (shifting of the disc upward due to the imbalance between the ganglion cell fibres coming from the superior and inferior retina). ${ }^{3}$

The heredity of the condition is not yet established, perhaps because it may easily be missed in family members of affected individuals, hence the lack of reports of the familial nature of the syndrome. Single clinical features like inferior crescent or transposition of the optic disc have been reported as geneti-

From the Institute of Ophthalmology of the University of Nijmegen, The Netherlands** and the Eye Clinic of the University of Milan at the Institute of Biomedical Science, San Paolo Hospital, Milan, Italy.*

The study was performed at the Institute of Ophthalmology of the University of Nijmegen.

Correspondence to: Ferdinando Bottoni MD, Via Andrea verga 4, 20144 Milano, Italy. 
cally determined with various inheritance patterns. ${ }^{4}$ On the other hand, Riise ${ }^{4}$ believes that the tilted disc syndrome can be familial, with a polymeric mode of inheritance similar to that of refraction anomalies.

In this study, we report on a family with tilted disc syndrome in three consecutive generations, the degree of expression being variable in the three patients. Peculiar fundus findings of interest as to the nasal ectasia were also observed.

\section{Case Report}

Case 1

A 40-year-old female patient (propositus, II-3) (Fig. 1) visited our outpatient department complaining of impaired vision for distance from childhood.

Visual acuity was $6 / 75$ with myopic astigmatism in both eyes (RE: $-5.5 /-2.0 \times 145$, LE: -6.25 / $-2.5 \times 20)$.

The intraocular pressures were normal.

Ophthalmoscopy demonstrated similar findings bilaterally: snowflakes degeneration in the far periphery; optic discs nasally tilted with inverse vessels emergence; a zone of elevated nerve fibres forming a prominent rim temporally (temporal supertraction) and no inferior crescent.

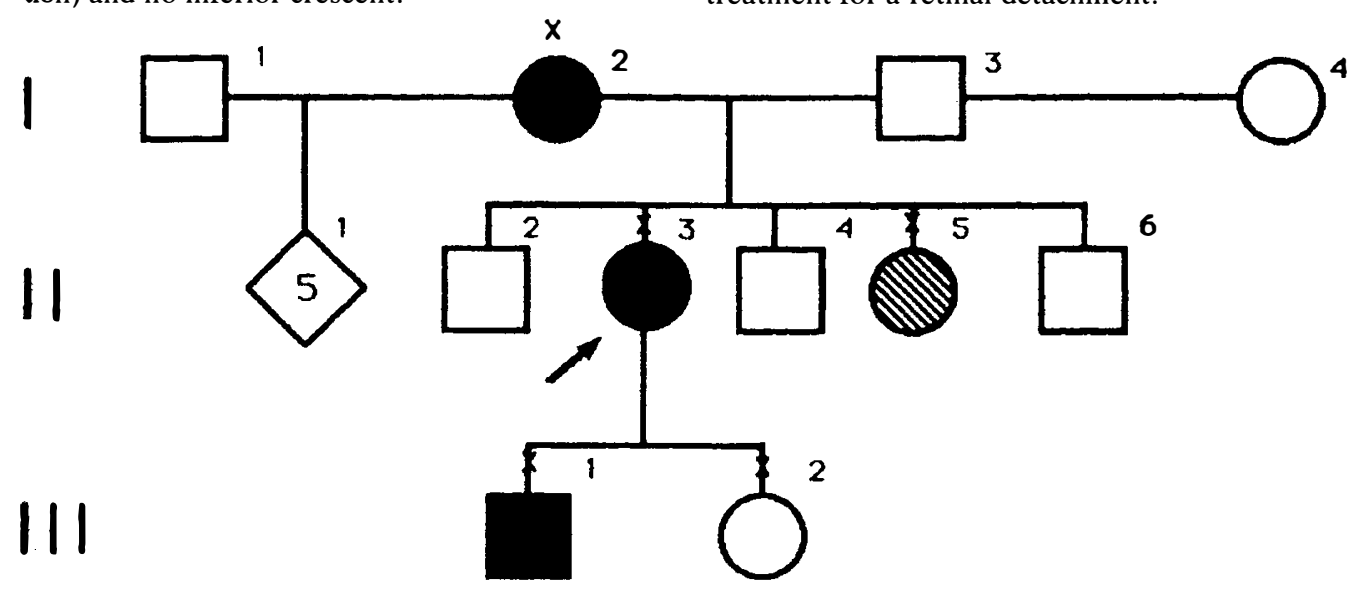

A deep, pale ectasia of the fundus, nasal and inferonasal to the optic disc, was centred by an atrophic scar which was pigmented in the right eye; linear hypopigmented streaks, radiating from the central scar, were found running parallel to the optic nerve margin in a circular pattern around the disc (Fig. 2).

Fluorescein angiography confirmed bilateral thinning of the retinal pigment epithelium and choroid inferonasally.

The remaining lesions, the atrophic scars and streaks, showed window defects but blockage of fluorescence corresponding to the pigment clump nasal to the disc in the right eye.

Kinetic Goldmann perimetry with small targets (I-2, I-1) revealed a bitemporal hemianopsia which did not respect the vertical boundary at the midline.

Ocular B-scan ultrasonography revealed that the antero-posterior diameters of the two globes were equal. By horizontal manoeuvres with the scanning probe, a posterior staphyloma was visible nasal and inferonasal to the optic nerve (Fig. 3).

Case 2

The 80 -year-old mother (I-2) of patient 1 , had been followed in our outpatient department since 1975 with degenerative myopia.

At age 50 years, the right eye underwent surgical treatment for a retinal detachment. 


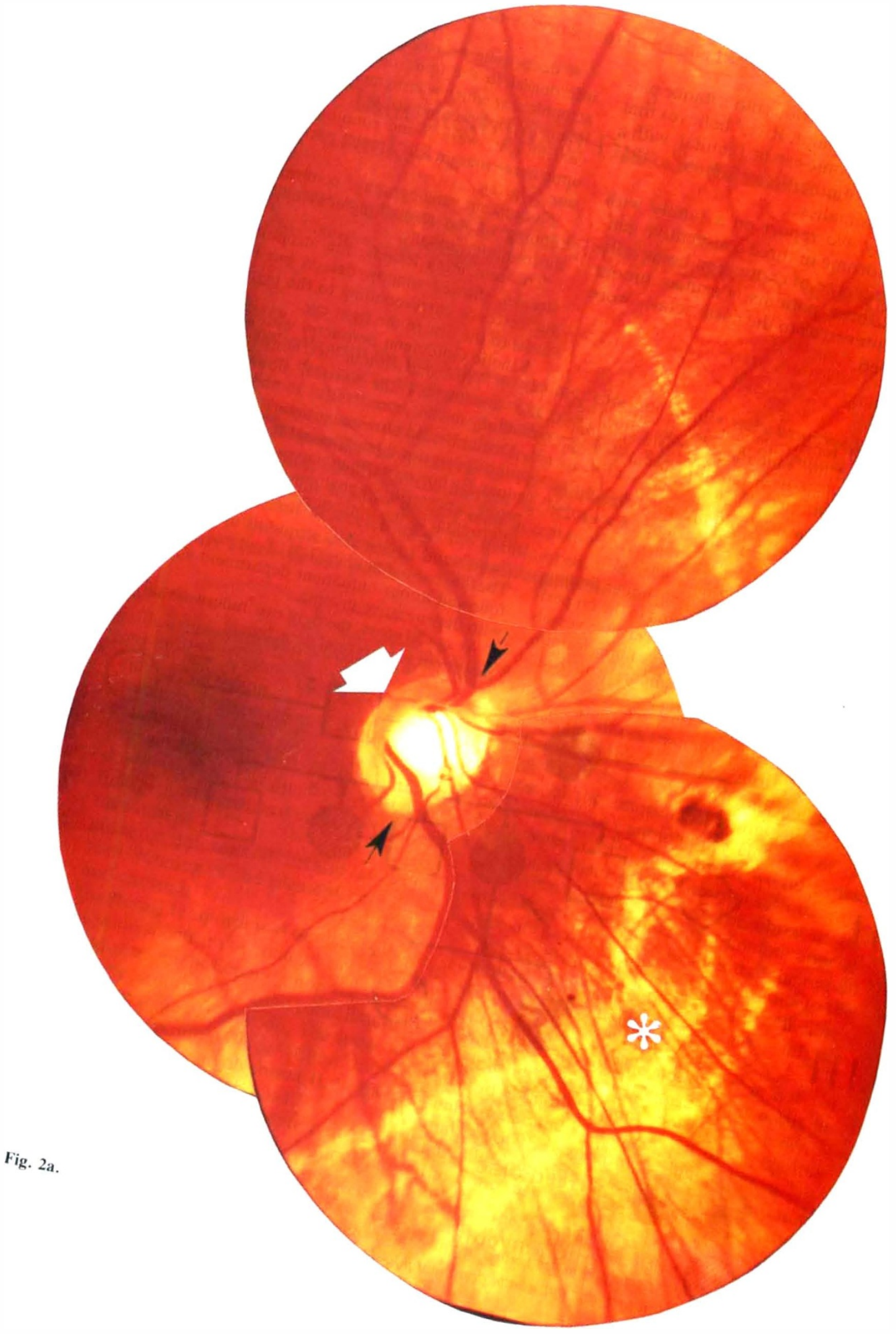




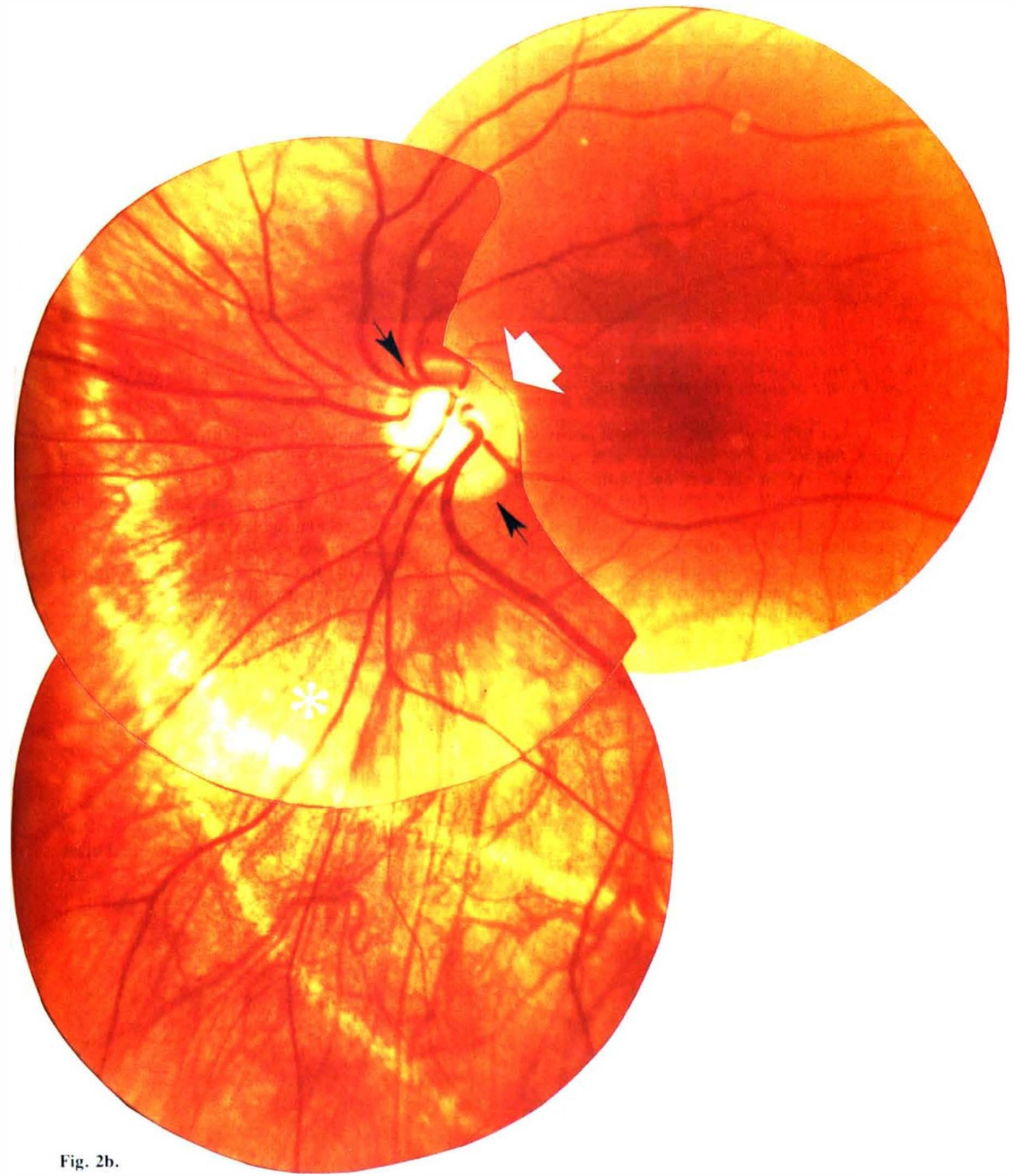

Fig. 2b.

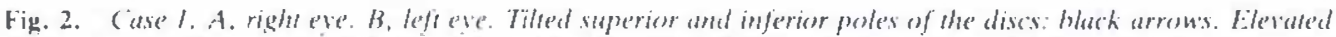

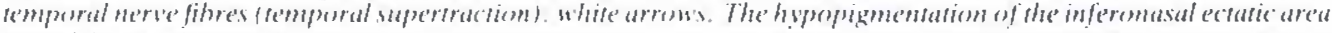

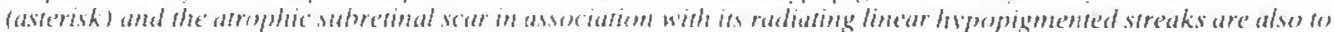
be noted. 


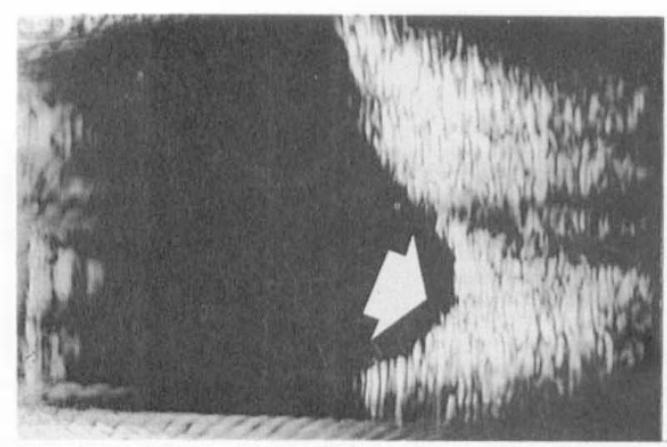

Fig. 3a.

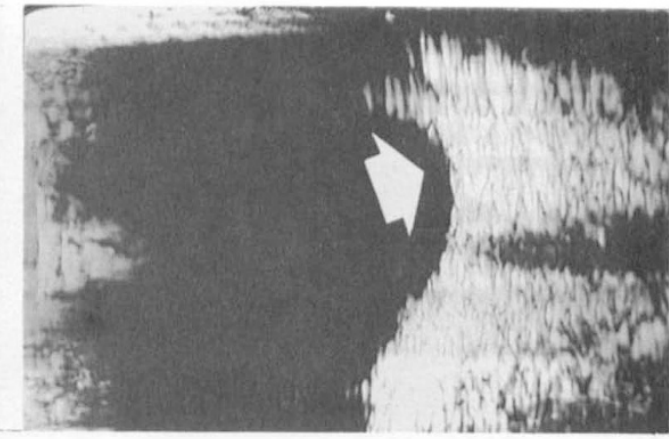

Fig. 3h.

Fig. 3. Case 1. A, right eye. B, left eye. Horizontal median section of both eyes by B-scan ultrasonography. Note irregular shape of globes from posterior inferonasal staphyloma (arrows).

At her last follow-up visit, her visual acuity was counting fingers in the right eye with a -15.0 sphere and $6 / 12$ in the left eye with a -10.0 / $-0.75 \times 100$.

Her intraocular pressures were normal.

Ophthalmoscopy of the right eye revealed patches of chorioretinal atrophy throughout the fundus with a posterior central staphyloma and a pale optic disc with no evidence of tilted disc syndrome. The left fundus displayed peripheral myopic chorioretinal degenerations and the tilted disc syndrome. Mild transposition of the optic disc without supertraction, inferior crescent and an hyperfluorescent area corresponding to the inferonasal ectasia were seen on fluorescein angiography (Fig. 4). B-scan ultrasonography confirmed the posterior staphyloma inferonasal to the optic disc.

\section{Case 3}

The 20-year-old son (III-1) of patient 1 , was examined by us.

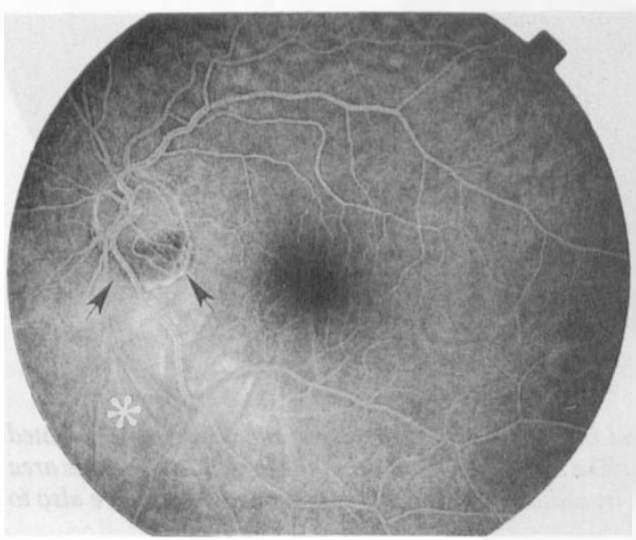

Fig. 4. Case 2, late phase fluorescein angiography of the left eye. Inferior crescent: black arrow. Inferonasal ectasia: asterisk.
His visual acuity was $6 / 9$ in both eyes with a myopic astigmatism (RE: $-6.0 /-2.25 \times 150$, LE: $-7.0 /-1.75 \times 30)$.

Ophthalmoscopy: the discs were tilted nasally with inverse vessel emergence, temporal supertraction and inferior crescents. Both eyes showed inferonasal ectasia, the left ectatic area being centered by a small subretinal atrophic scar (Fig. 5a).

Fluorescein angiography disclosed the hypoplastic retinal pigment epithelium and choroid in the inferonasal quadrants; the small scar showed no evidence of neovascular ingrowth (Fig. 5b).

A relative bitemporal defect was detected on Goldmann kinetic perimetry.

B-scan ultrasonography demonstrated the presence of bilateral ectasia, inferonasal to the optic disc.

Other family members

The 45 -year-old sister (II-5) of patient 1 , had been

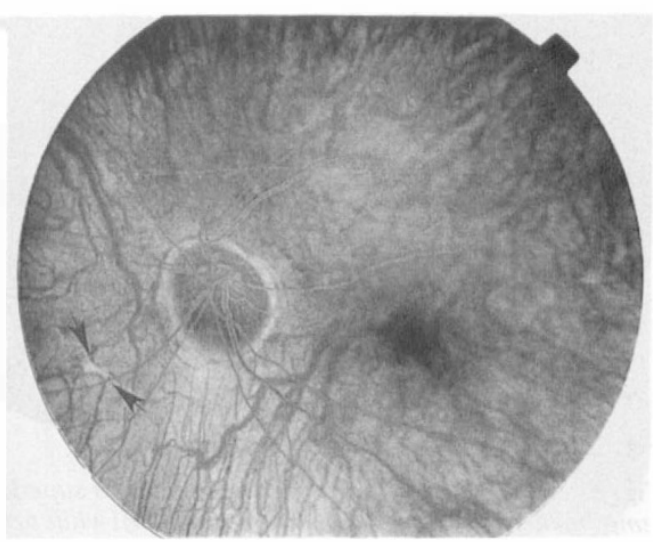

Fig. 5. Case 3, late phase fluorescein angiography of the left eye. Tilted disc, inverse vessels emergence, temporal supertraction and inferior crescent are to be noted. Small subretinal atrophic scar at the centre of the inferonasal ectatic area: black arrows. 
followed in our outpatient department since 1975 with pathologic myopia.

At age 27 years, a peripheral retinal defect in the right eye was successfully treated by Argon laser photocoagulation.

At her last examination, her visual acuity was $6 / 60$ in the right with a -21.0 sphere and $6 / 21$ in the left eye with -22.0 sphere. Ophthalmoscopy demonstrated patches of chorioretinal atrophy extending across the fundus bilaterally with a Fuch's spot at the right macula. There was no evidence of tilted disc syndrome in either eye.

\section{Discussion}

Abnormalities of the fundus in our three patients show the major signs of the tilted disc syndrome, with the exception of patient 1 who lacked the crescent inferior to the disc. Cases 1 and 3 had both eyes involved, while only the left eye was affected in case 2. Myopic astigmatism with oblique cylinders, as previously reported, ${ }^{1-4}$ was seen in each patient, the visual acuity being moderately reduced even with the best correction. This might be due to the Stiles-Crawford effect as the photoreceptors stand obliquely in relation to the visual axis. ${ }^{4}$

Reduced visual acuity in association with bitemporal depression of the visual field corresponding to the inferonasal ectasia can suggest a lesion near the optic chiasm. Recognition of the fundus abnormalities, possible improvement of the field defect with proper refractive correction ${ }^{4}$ and lack of the vertical boundary at the midline of the visual field should obviate the need for major neurological work-up.

The diagnosis of tilted disc syndrome by examination in three consecutive generations demonstrates the dominant mode of inheritance of the disease in the present pedigree, the expressivity being variable in our three patients.

Patient 1 showed bilateral inferonasal ectasia which was centered by atrophic scars, the right one resembling Fuch's spot. The hypopigmented streaks radiating from the central scar bilaterally had ophthalmoscopic and fluoro-angiographic features of breaks in Bruch's membrane (i.e., lacquer crack lesions) with a typical shape.

The changes of Fuch's spot and lacquer cracks are common findings in degenerative myopia $^{5,6}$ and are likely to be caused by mechanical stretching of the retina and choroid within the posterior staphyloma.?

Unlike myopic lacquer cracks, which show a reticular pattern at the posterior pole ${ }^{8}$ the lesions in our patient were very similar to the ones usually seen in traumatic tears of Bruch's membrane, presenting a circular pattern around the disc. ${ }^{8}$

Moreover, the specular eccentricity of the fracture distribution, usually temporal to the optic nerve in traumatic lacquer cracks and nasal to the optic nerve in our case should be noted. The mechanical stretching of the ectatic area and its abnormal inferonasal location with respect to the optic nerve may have produced the linear-like pattern of the lacquer cracks in our patient.

\section{References}

${ }^{1}$ Apple DJ, Rabb MF, Walsh PM: Congenital anomalies of the optic disc. Surv Ophthalmol 1982, JulAug, 27(1): 3-41.

${ }^{2}$ Dorrell D: The tilted disc. Br J Ophthalmol 1978, 62: 16-20.

${ }^{3}$ Giuffre' G: Hypothesis on the pathogenesis of the papillary dysversion syndrome. J Fr Ophtalmol 1985, 8: 565-72.

${ }^{4}$ Riise D: The nasal fundus ectasia. Acta Ophthalmol 1975, Suppl 126: 1-108.

${ }^{5}$ Gass JDM: Pathogenesis of disciform detachment of the neuroepithelium. VI. Disciform detachment secondary to heredodegenerative, neoplastic and traumatic lesions of the choroid. Am J Ophthalmol 1967, 63: 689-711

${ }^{6}$ Curtin BJ, Karlin DB: Axial length measurements and fundus changes of the myopic eye. Am J Ophthalmol 1971, 71: 42-53.

${ }^{7}$ Curtin BJ: The etiology of myopia. In The Myopias. Philadelphia, Harper \& Row, pp 61-64, 1985.

${ }^{8}$ Pruett RC, Weiter JJ, Goldstein RB: Myopic cracks, angioid streaks and traumatic tears in Bruch's membrane. Am J Ophthalmol 1987, 103: 537-43. 